\title{
Characteristic Trend Analysis of Cancer Patients Hospitalized in Shanxi Tumor Hospital for the First Time during 2001 and 2010
}

\author{
Wen-Li Zhang ${ }^{1}$, Yan Wang ${ }^{2}$, Cun-Zhi Han ${ }^{2 *}$
}

\begin{abstract}
To observe and analyze the characteristic trend of cancer patients hospitalized for the first time in Shanxi Tumor Hospital from 2001 to 2010, clinical data including case number, age, gender, and frequency of different tumor occurrences were collected and statistically analyzed. Results: (i) From 2001 to 2010, the number of cancer patients hospitalized for the first time increased by 1.3-fold; (ii) The patient overall average age also increased from 51.8 to 54.4, for males from 55.5 to 58.7 and females from 48.4 to 51.1, respectively. (iii) Male patients accounted for $43-48 \%$ and females accounted for $52-57 \%$ of the total. The percentage of female patients was higher than that of male patients in every year and showed an upward trend over the years, while that of the males showed a downward trend $\left(\chi^{2}=7.031, p=0.008\right.$ ); (iv) Among the top 6 most common cancers, lung, cervical, esophageal, colorectal and breast cancers tended to increase over the years $(p<0.05)$, but not gastric cancer $(p=\mathbf{0 . 4 2 3})$. Conclusions: (i) The number of cancer patients hospitalized for the first time during the past 10 years increased year by year, and was higher for female than male; (ii) the average age of patients increased year after year and was greater for male than female; (iii) the number of patients with lung cancer, cervical cancer, esophageal cancer, colorectal cancer and breast cancer increased over years.
\end{abstract}

Keywords: Cancer inpatients - trend analysis - age - gender - Shanxi, China

Asian Pac J Cancer Prev, 16 (9), 3673-3676

\section{Introduction}

Hospital registries collect, manage, analyze, and report information on cancer patients who are newly diagnosed and/or treated, and provide useful data to evaluate patient care within the hospital. This type of data may be focused on the care of cancer patients. The data can be used in studies that compare patterns of care among providers, population subsets, or geographic regions.

Shanxi Tumor Hospital is a large and nationally wellknown comprehensive cancer center specialized in clinical management and applied research serving cancer patients from Shanxi Province and its surrounding Northern China provinces. In this paper, we analyzed the trend of the number, age, gender and different cancer occurrences of cancer patients hospitalized in our hospital for the first time between 2001 and 2010 .

\section{Materials and Methods}

This study was approved by the Ethics Committee of Shanxi Tumor Hospital. No written consent form was obtained since we used the registry data. No written consent was given by the patients for their information to be stored in the database and used for research.

The clinical data such as case number, age, gender, and trend of different tumor occurrence of cancer patients who was hospitalized for the first time in this Hospital for the past 10 years were collected and statistically analyzed. The cancer incidence rates from 2001 to 2010 were calculated with reference to the new cancer cases identified in Shanxi Tumor Hospital. Linear regression analysis was utilized to depict the relationship between the first visiting year and the average age of the patients. The Chi-square test was used to depict yearly-based trending. All statistical tests were 2 sided and considered statistically significant for $p \leq 0.05$.

\section{Results}

Number of cancer patients hospitalized in Shanxi Tumor Hospital for the first time during January 2001 and December 2010

Among the total of 156,433 cancer patients hospitalized from 2001 to $2010,117,738$ were hospitalized for the first time. Their number was slowly increased from 2001 to 2007, and thereafter rapidly increased, by 1.3 -fold during 10 years. The results are shown in Figure 1. 
Age of cancer patients hospitalized in Shanxi Tumor Hospital for the first time during January 2001 and December 2010

Trend analysis of the age of the patients hospitalized for the time from 2001 to 2010 showed that: $i$ ) their average age at hospitalization was increased year after year for both male and female; $i$ ) compared with the overall average ages from 51.82 to 54.39 , the average age of male patients was 3.5-4.5 years higher, and that of female patients was 3-3.5 years lower; iii) the average age of the male patients was 6-7 years higher than that of the female patients; $i v$ ) The regression analysis of the scatter plot of the average age versus the year of first hospitalization showed a linear relationship with a regression equation of $\mathrm{Y}=0.281 \mathrm{X}-510.611(p<0.05)$, meaning that for every additional year, patients' average age increased by 0.281 years old as shown in Table 1.

Gender of cancer patients hospitalized in Shanxi Tumor Hospital for the first time during January 2001 and December 2010

Trend analysis of the gender of the patients hospitalized for the first time in the 10 years from 2001 to 2010 showed that: $i)$ the percentage of female patients hospitalized for the first time was higher than that of male patients during the 10 years; $i$ ) the percentage of male patients hospitalized for the first time was $43 \%-48 \%$, while that of female patients was $52 \%-57 \%$; iii) $\chi^{2}$ trend analysis indicated that the percentage of male patients hospitalized for the first time showed a downward trend over the years, while that of the females showed an upward trend $\left(\chi^{2}=7.031, p=0.008\right)$. The results are shown in Figures 3 and 4.
Analysis of common cancers in patients hospitalized in Shanxi Tumor Hospital for the first time during January 2001 and December 2010

Trend analysis of common cancers among patients hospitalized for the time from 2001 to 2010 showed that i) patients with lung, cervical and esophageal cancers ranked the first, second and third place, and their numbers increased by almost 2-, 3-, and 2-fold from 2001 to 2010, respectively; $i$ ) during the 10 years, patients with breast, gastric and colorectal cancers increased nearly 3-fold and ranked the fourth, fifth and sixth place, respectively; iii) during the 10 years, although only a few patients with prostate and endometrial cancers were hospitalized, their number increased dramatically by 9 - and 7 -fold,

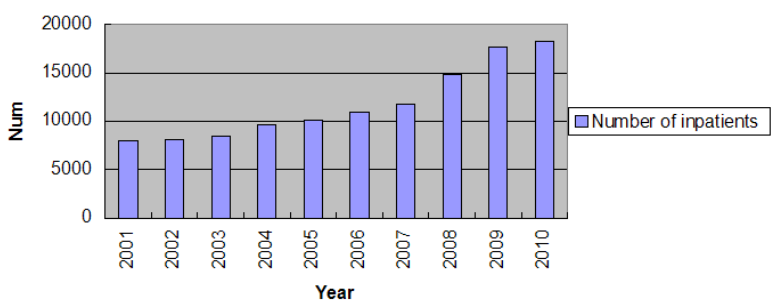

Figure 1. Number of Cancer Patients Hospitalized for the First Time during Jan. 2001 and Dec 2010

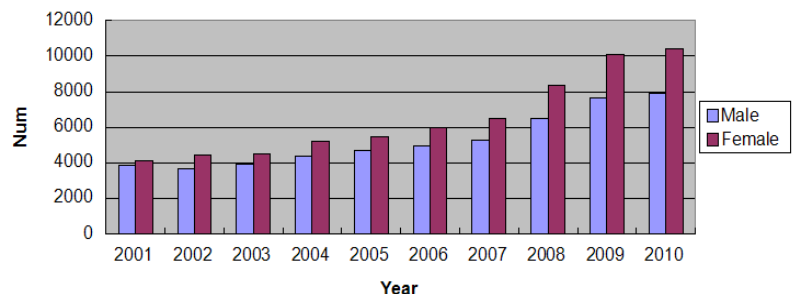

Figure 2. Gender of Cancer Patients Hospitalized for the First Time during Jan. 2001 and Dec 2010

Table 1. Age for the First Time Hospitalized Patients During 2001 and 2010

\begin{tabular}{lcccccccccc}
\hline Year & 2001 & 2002 & 2003 & 2004 & 2005 & 2006 & 2007 & 2008 & 2009 & 2010 \\
\hline Average(yr) & 51.82 & 51.84 & 52.48 & 52.75 & 52.79 & 52.91 & 53.22 & 53.63 & 54.12 & 54.39 \\
Male & 55.54 & 55.42 & 56.49 & 56.87 & 57.42 & 57.42 & 57.43 & 57.9 & 58.39 & 58.74 \\
Female & 48.38 & 48.88 & 49.29 & 49.29 & 49.14 & 49.14 & 49.8 & 50.32 & 50.88 & 51.09 \\
\hline
\end{tabular}

Table 2. The Number of Common Cancer Patients among the First Time Hospitalized Cancer Patients during Jan 2001 and Dec 2010

\begin{tabular}{|c|c|c|c|c|c|c|c|c|c|c|}
\hline Group N(\%) & 2001 & 2002 & 2003 & 2004 & 2005 & 2006 & 2007 & 2008 & 2009 & 2010 \\
\hline ancer & 11 & 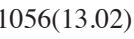 & ) & 117 & & $.27)$ & & $1838(12.37)$ & $2167(12.24)$ & 2153(11.78) \\
\hline Cervical cancer & $674(8.47)$ & $792(9.76)$ & $798(9.49)$ & $880(9.19)$ & $966(9.53)$ & $1011(9.27)$ & $1079(9.15)$ & $1523(10.25)$ & $1832(10.35)$ & $1817(9.94)$ \\
\hline Esophageal cancer & $682(8.57)$ & $625(7.71)$ & $712(8.46)$ & $764(7.98)$ & $817(8.06)$ & $839(7.69)$ & $904(7.67)$ & $1110(7.47)$ & $1213(6.85)$ & $1336(7.31)$ \\
\hline Colorectal cancer & $484(6.08)$ & $455(5.61)$ & $457(5.43)$ & $574(5.99)$ & $668(4.72)$ & $685(6.28)$ & $740(6.28)$ & $847(5.7)$ & $1092(6.17)$ & $1161(6.35)$ \\
\hline Breast cancer & $386(4.85)$ & $397(4.89)$ & $395(4.7)$ & $460(4.8)$ & $550(5.42)$ & $665(6.1)$ & $682(5.78)$ & $837(5.64)$ & $1022(5.77)$ & $1089(5.96)$ \\
\hline Gastric cancer & $442(5.55)$ & $434(5.35)$ & $510(6.06)$ & $543(5.67)$ & $609(6.01)$ & $603(5.53)$ & $708(6.01)$ & $915(6.16)$ & $1006(5.68)$ & $1027(5.62)$ \\
\hline Malignant lymphoma & $238(2.99)$ & $189(2.33)$ & $190(2.26)$ & $247(2.58)$ & $248(2.45)$ & $227(2.08)$ & $241(2.04)$ & 297(2) & $323(1.82)$ & $364(1.99)$ \\
\hline Hepatic carcinoma & $173(2.17)$ & $182(2.24)$ & $182(2.16)$ & $171(1.79)$ & $186(1.83)$ & 193(1.77) & 201(1.7) & $253(1.7)$ & $328(1.85)$ & $334(1.83)$ \\
\hline Thyroid & $123(1.55)$ & $151(1.86)$ & $124(1.47)$ & 191(1.99) & 191(1.88) & $172(1.58)$ & $182(1.54)$ & $212(1.43)$ & $274(1.55)$ & $317(1.73)$ \\
\hline Ovarian cancer & $114(1.43)$ & $109(1.34)$ & $119(1.41)$ & $146(1.52)$ & $153(1.51)$ & $167(1.53)$ & $176(1.49)$ & $214(1.44)$ & $253(1.43)$ & $268(1.47)$ \\
\hline Endometrial cancer & $38(0.48)$ & $26(0.32)$ & $69(0.82)$ & $78(0.81)$ & $107(1.06)$ & $131(1.2)$ & $127(1.08)$ & $150(1.01)$ & $181(1.02)$ & $217(1.19)$ \\
\hline Kidney cancer & $49(0.62)$ & $56(0.69)$ & $57(0.68)$ & $74(0.77)$ & $89(0.88)$ & $113(1.04)$ & $99(0.84)$ & $128(0.86)$ & $156(0.88)$ & $176(0.96)$ \\
\hline Bone cancer & $144(1.81)$ & $56(0.69)$ & $71(0.84)$ & $60(0.63)$ & $60(0.59)$ & $45(0.41)$ & $32(0.27)$ & $31(0.21)$ & $50(0.28)$ & $103(0.56)$ \\
\hline Gallbladder & $45(0.57)$ & $33(0.41)$ & $37(0.44)$ & $43(0.45)$ & $43(0.42)$ & $42(0.39)$ & $56(0.47)$ & $79(0.53)$ & $115(0.65)$ & $98(0.54)$ \\
\hline Pancreatic cancer & $28(0.35)$ & $23(0.28)$ & $21(0.25)$ & $30(0.31)$ & $27(0.27)$ & $44(0.4)$ & $39(0.33)$ & $51(0.34)$ & $74(0.42)$ & $76(0.42)$ \\
\hline Brain tumor & $42(0.53)$ & $32(0.39)$ & $48(0.57)$ & $51(0.53)$ & $36(0.35)$ & $55(0.5)$ & $47(0.4)$ & $74(0.5)$ & $76(0.43)$ & $73(0.4)$ \\
\hline Leukemia & $30(0.38)$ & $28(0.35)$ & $18(0.21)$ & $35(0.37)$ & $34(0.34)$ & $28(0.26)$ & $23(0.2)$ & $29(0.2)$ & $90(0.51)$ & $61(0.33)$ \\
\hline Prostate cancer & $6(0.08)$ & $10(0.12)$ & $15(0.18)$ & $13(0.14)$ & $10(0.1)$ & $13(0.12)$ & $16(0.14)$ & $36(0.24)$ & $69(0.39)$ & $58(0.32)$ \\
\hline $\begin{array}{l}\text { Endometrial } \\
\text { adenocarcinoma }\end{array}$ & 13(0.16) & $7(0.09)$ & $21(0.25)$ & $20(0.21)$ & $21(0.21)$ & $2(0.02)$ & $4(0.03)$ & $13(0.09)$ & $6(0.03)$ & $7(0.04)$ \\
\hline
\end{tabular}

*Note: The order was based on the data of 2010 
Table 3. Trend Analysis of the Common Cancers in the First Time Hospitalized Cancer Patients during January 2001 and December 2010

\begin{tabular}{lcccccc}
\hline Group & Lung cancer & Cervical cancer & Esophageal cancer & Colorectal cancer & Breast cancer & Gastric cancer \\
\hline$X^{2}$ & 5.738 & 4.113 & 6.303 & 5.114 & 6.392 & 0.641 \\
$p$ & 0.017 & 0.043 & 0.012 & 0.024 & 0.001 & 0.423 \\
\hline
\end{tabular}

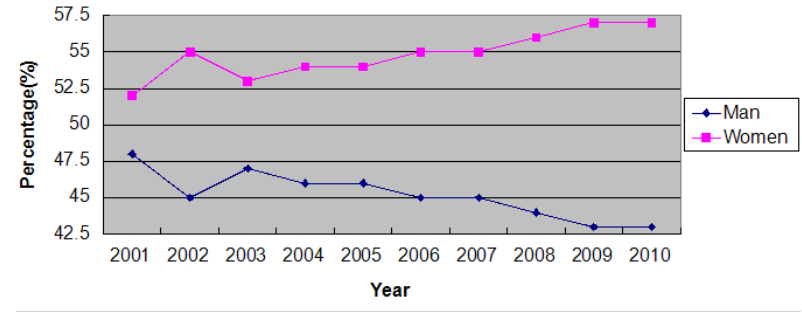

Figure 3. The Percentage of Common Cancer Patients among Cancer Patients Hospitalized for the First Time during Jan 2001 and Dec 2010

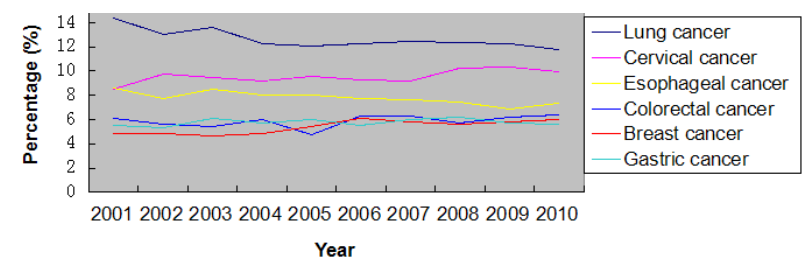

Figure 4. Analysis of the First Six Common Cancers in Cancer Patients Hospitalized for the First Time during Jan. 2001 and Dec 2010

respectively; in addition, the number of patients with liver, thyroid, ovarian, gallbladder, pancreatic and bladder cancers increased more than 2-fold while the number of patients with endometrial cancer, bone cancer, brain tumors, blood disease and liver cancer showed a downward trend. $\chi^{2}$ analysis of patients with the first six common cancers hospitalized for the first time during January 2001 and December 2010 showed that the number of patients with lung, cervical, esophageal, colorectal and breast cancers increased over the 10 years, $(p<0.05)$, while that of patients with gastric cancer was not changed $(p=0.423)$. The results are shown in Tables 2 and 3 and in Figure 5.

\section{Discussion}

This study indicated that cancer patients hospitalized for the first time in Shanxi Tumor Hospital increased by 1.3 -fold during the 10 years from 2001-2010. The number of patients increased slowly until 2007 and then rapidly increased. The following factors could attribute the phenomena: $i$ ) The hospital has been expanded since early May of 2008 on. A new surgical building with more than 700 beds was placed in use, which increased the hospital's capacity for cancer patient admissions; $i$ ) Central Government implemented new health insurance policies, which covers the provincial health insurance, municipal health insurance, residents health insurance, railway company health insurance, especially the new rural cooperative medical insurance policy, which promoted patients' willingness to visit a hospital. In addition, this new policy allowed patients to be freely admitted by a hospital at their will; iii) Shanxi Tumor Hospital is a prestigious specialized hospital for cancer patients in Shanxi Province and the only hospital which could treat patients using comprehensive measures including surgery, radiotherapy, chemotherapy, interventional therapy, targeted therapy, biological therapy, traditional Chinese medicine and hospice. In addition, the hospital is a multifunctional modern cancer hospital with state-of-the-art facilities, as well as skilled health care professionals, and could provide consecutive service of pre-, within and post hospitalization. All the above attracts patients to come for clinical management of their diseases.

The survey indicated that the average ages of patients hospitalized for the first time showed an increased trend year by year. This is mainly due to the improved living standards, enhanced prevention and health awareness, regular medical examination, early care and early prevention, all of which postpone the age at tumor occurrence. The survey also showed that male patients accounted for $43 \%-48 \%$ of the total hospitalized patients, and female accounted for $52 \%-57 \%$, significantly higher than that of the male. This is possibly due to the following reasons: $i$ ) The morbidity and mortality of cervical cancer in women in Shanxi ranks the first in China (Zhao et al., 2004; Zen et al., 2004), even though the fact that the morbidity and mortality of cervical cancer has decreased slightly for the recent years because the provincial government has carried out a large scale census for cervical cancer and developed early detection and treatment measures (Rong et al., 2002). ii) Over the past decade, lifestyle has been increasingly westernized, incidence increase of breast cancer in females is accelerated, especially in middle-aged women, forming a new incidence peak ( $\mathrm{Ji}$ et al., 2011). But Chen et al. reported that cancer incidence rates for males were higher than those for females (Chen et al., 2014).

This study showed that patients with lung cancer had been in the first place for the 10 years. Lung cancer is one of the typical diseases closely related to environmental factors and lifestyles. In addition to smoking, the most recognized causative factor, Shanxi is the most important chemical and energy industry base in China, coal and coke industry have caused serious environmental pollution. In addition, the increased heating systems and automobiles may also be an important factor leading to the rise of lung cancer (Chen et al., 2010; Chen et al., 2011; Jiang et al., 2012). Numbers of patients with cervical and esophageal cancers also increased during the 10 years. Shanxi Province is located in the Taihang Mountain area, which is one with high incidence of esophageal and cervical cancers. The incidence of cervical cancer is related to many factors. Among them, HPV infection is an important one (Sheng et al., 2003; Guan et al., 2006; Qiao et al., 2007; Li et al., 2013). Esophageal cancer is a 
common malignant tumors, the morbidity and mortality of it in China are one of the highest worldwide and possibly associated with external environmental factors, unhealthy lifestyles, genetics and gene polymorphisms (Chen et al., 2003; Wang et al., 2011). The number of patients hospitalized for the first time due to breast and colon cancers showed a gradual upward trend during the 10 years. These cancers are caused by many factors and complex mechanisms. Owing to the westernization of life behavior and diet structure for the recent years, the incidence of breast and colorectal cancers showed an upward trend (Kuriyama et al., 2005; Pei et al., 2008). Therefore, enhancing the awareness of the risk factors for malignant tumors, improving the recognition of colorectal and breast cancers, and reasonable diet and nutrition education are important measures to prevent the occurrence of colon and breast cancers.

\section{Acknowledgements}

This research is supported by Department of Information, Shanxi Cancer Hospital, Taiyuan, Shanxi, China. Drs. Zhong-Qian Li and Zivjena Vucetic of Fujirebio Diagnostics, Inc. (Malvern, PA, USA) are appreciated for their editing comments. The study protocol was approved by the Ethics Committee of the ShanXi Cancer Hospital, Taiyuan, China. Before the experiments, the subjects were informed of the objectives, requirements and procedures of the experiments. All gave informed written consent to participate in the study.

\section{References}

Rong SD, Chen W, Wu LY, et al (2002). Risk factors for cervical cancer in Xianghuan county, Shanxi province. Chin J Prev Med, 36, 42-6.

Chen W, Sun XD, Fan JH, et al (2003). Variation of risk factors for esophageal cancer in Linxian, China. Cancer Res Clin, 15, 5-7.

Chen WQ, Zhang SW, Zou XN, et al (2010). An analysis of lung cancer mortality during 2004-2005 in China. Chin J Prev Med, 44, 378-82.

Chen RJ, Zhang JL , Han HD (2011). Review of epidemiological studies of air pollution and lung cancer in China. J Hygiene Res, 40, 243-5.

Chen PL, Zhao T, Feng R, et al (2014). Patterns and trends with cancer incidence and mortality rates reported by the China National cancer registry. Asian Pac J Cancer Prev, 15, 6327-32.

Guan T, Zhang ZW, Xie YF, et al (2006). Relationship between human papillomavirus genotype and cervical intraepithelial neoplasia. Maternal Child Health Care China, 21, 960-3.

$\mathrm{http}: / / \mathrm{www} . c a n c e r . o r g / c a n c e r / c a n c e r b a s i c s / c a n c e r-s u r v e i l l a n c e-$ programs-and-registries-in-the-united-states. Accessed on October 23, 2013.

http://www.dukeraleighhospital.org/healthservices/cancercenter/about/2012-reports/cancer-registry. Accessed on October 23, 2013.

Ji FM , Ha N (2011). Epidemiology of breast cancer in female from 1996-2007. World Health Dig, 8.

Jiang HC, Shen F, Yang YB (2012). Statistic analysis of inpatients with lung cancer during 2003-2010. Chin J Hospital Statistics, 19, 239-41.
Kuriyama S, Tsubono Y, Hozawa A, et al (2005). Obesity and risk of cancer in Japan. Int J Cancer, 113, 148-57.

Li J, Huang R, Schmidt JE, Qiao YL (2013). Epidemiological features of Human Papillomavirus (HPV) infection among women living in Mainland China. Asian Pac J Cancer Prev, 14, 4015-23.

Pei GG, Fu L , Cui YL (2008). Risk factor meta-analysis of Chinese women with breast cancer. Maternal \& Child Health Care China, 23, 2650-2.

Qiao YL (2007). Epidemiological studies and status quo of Chinese women with papillomavirus infection and cervical cancer and prospects for preventable vaccine. Chinese $J$ Epidemiol, 28, 937-40.

Sheng YH, Chen F, Huang MN, et al (2003). Population-based study of human papillomavirus infection in high-risk area for cervical cancer in Shanxi Province, China. Acta Acad Med Sin, 25, 382-5.

Wang XZ, Yuan FM, Wang F, et al (2011). Analysis of incidence of esophageal cancer in Yangcheng County during 1999 and 2007. China Cancer, 20, 419-21.

Zhao FG, Qiao YL(2004). Advance in etiology of cervical cancer. Oncol Prog, 2, 39-42.

Zen ZP, Chen F, Liu B, et al (2004). Risk factors for cervical cancer in Yangcheng county, Shanxi province. Cancer Res Prev Treat, 31, 178-81. 